Document downloaded from:

http://hdl.handle.net/10251/73712

This paper must be cited as:

Luis Fernando Alarcón; Diego Acuña; Sven Diethelm; Pellicer, E. (2016). Strategies for improving safety performance in construction firms. Accident Analysis and Prevention. 94:107-118. doi:10.1016/j.aap.2016.05.021.

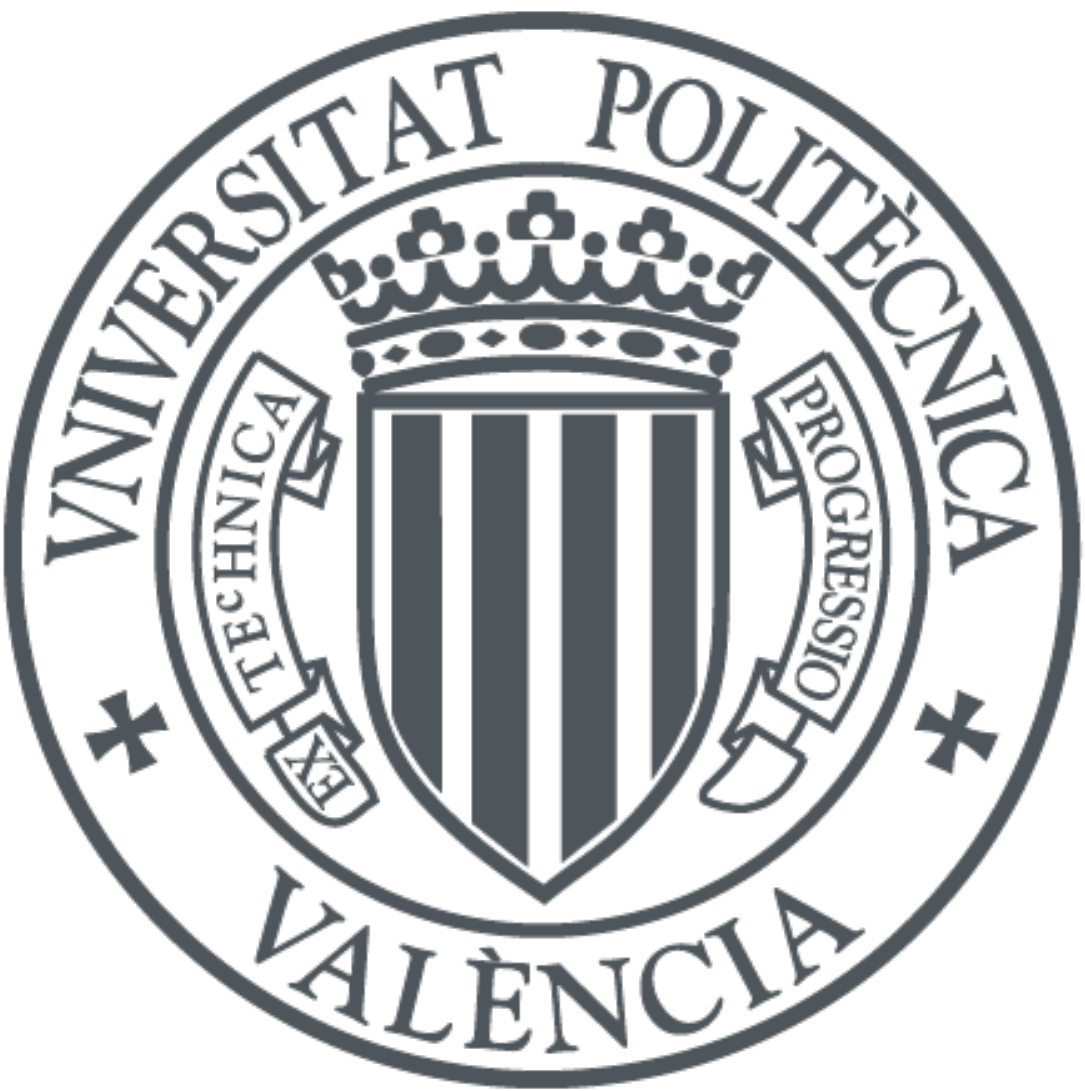

The final publication is available at

http://dx.doi.org/10.1016/j.aap.2016.05.021

Copyright Elsevier

Additional Information 


\title{
STRATEGIES FOR IMPROVING SAFETY PERFORMANCE IN
} CONSTRUCTION FIRMS

\author{
Luis Fernando Alarcón ${ }^{a}$, Diego Acuña ${ }^{b}$, Sven Diethelm ${ }^{c}$, and Eugenio Pellicer ${ }^{d}$ \\ a Pontificia Universidad Católica de Chile, Vicuña Mackenna 4860, Santiago, Chile, e-mail: \\ lalarcon@ing.puc.cl \\ ${ }^{\text {b }}$ Pontificia Universidad Católica de Chile, Vicuña Mackenna 4860, Santiago, Chile, e-mail: \\ deacuna@puc.cl \\ c Centro de Excelencia en Gestión de Producción (GEPUC), Pontificia Universidad Católica de Chile, e- \\ mail: sdiethel@gepuc.cl \\ ${ }^{\mathrm{d}}$ Corresponding Author: School of Civil Engineering, Universitat Politècnica de València, Camino de Vera \\ s/n, 46022 Valencia (Spain), phone \#: +34.963.879.562; fax \#: +34.963.877.569; e-mail: \\ pellicer@upv.es
}

\section{Abstract}

Over the years many prevention management practices have been implemented to prevent and mitigate accidents at the construction site. However, there is little evidence of the effectiveness of individual or combined practices used by companies to manage occupational health and safety issues. The authors selected a sample of 1,180 construction firms and 221 individual practices applied in these companies to analyze their effectiveness reducing injury rates over a period of four years in Chile. Different methods were used to study this massive database including: visual analyses of graphical information, statistical analyses and classification techniques. Results showed that practices related to safety incentives and rewards are the most effective from the accident rate viewpoint, even though they are seldom used by companies; on the other hand, practices related to accidents and incidents investigation had a slight negative impact on the accident rate because they are frequently used as a reactive 
measure. In general, the higher the percentage of prevention practices implemented in a strategy, the lower the accident rate. However, the analysis of the combined effect of prevention practices indicated that the choice of the right combination of practices was more important than just the number of practices implemented.

Keywords: accident rate; Chile; construction company; prevention practice; safety management; strategy.

\section{Introduction}

Occupational safety and health have always been sensitive issues in the construction industry, particularly considering its high number of accidents (Hallowell, 2012; Pellicer et al., 2014). These accidents not only affect the health of workers, but also the future lives of entire families (Hinze, 2002a). They are also a source of losses for construction companies (Waehrer et al., 2007; Pellicer et al., 2014). Any contribution to help reduce occupational accidents in the construction industry can be considered worthy.

Throughout the years, the occurrence of accidents has gone from being considered a random phenomenon (Greenwood and Woods, 1919) to being the result of a series of factors that are possible to determine and control; there are many sources, at the individual and organizational level, that can cause accidents (Bird and Germain, 1985). Razuri et al. (2007) suggested that a combination of practices generates an incremental contribution of safety performance. Hence, prevention has become multifocal, meaning that there is no single formula to prevent accidents, but rather efforts or strategies to cover multiple areas of work. Therefore, detecting the best performing combinations of practices or strategies for different sizes of companies with different needs is a promising field; this is the point of departure of this research. 
In order to pursue this exploration further, the authors contacted the Safety Mutual of the Chilean Chamber of Construction; this a non-profit organization that provides medical insurance and technical assistance on safety management to companies in all types of industries. The authors, working with the Safety Mutual of the Chilean Chamber of Construction, selected a data sample of more than one thousand construction firms, and two hundred individual safety practices applied in these companies, so as to analyze their effectiveness in increasing safety performance over a period of four years. The analysis of safety performance (specifically the accident rate) and the implemented safety practices allows the identification of combined practices (strategies), and the selection of analysis techniques that have the potential to support the design of safety management strategies in the near future.

In the context of this research, a safety practice is a managerial process that implements one or more tools and techniques aiming to increase the occupational safety of the employees in a systematic way (Vinodkumar \& Bhasi, 2010; Bridi et al., 2013). These safety practices can lead to a safety culture in the organization, where collective behaviors of people become a pattern (Fung et al., 2005). From this research viewpoint, safety performance is measured using the accident rate, since it is a quantitative, reliable and common indicator (Vinodkumar \& Bhasi, 2010; Hallowell et al., 2013; Wachter \& Yorio, 2014). This indicator measures only those incidents that turn into injures or fatalities of workers (accidents). According to the Chilean law, information regarding accidents, and therefore the accident rates, is obtained directly from the official occupational accident report submitted to the Safety Mutual, which is considered to be a reliable source of information.

The rest of the paper is organized as follows. First, a literature review of safety management practices is carried out, and the knowledge gap is identified. Later, the research method is explained. This section is followed by a description and discussion of the results, considering different analysis techniques. Finally, the main contributions, limitations and future research are highlighted in the Conclusions section. 


\section{Literature Review}

Accidents happen in spite of the efforts that are done to prevent them. Knowing the underlying causes of accidents would allow attacking the root of this problem. Several authors have proposed different theories to predict their occurrence. First, Greenwood and Woods (1919) proposed the theory of accident-proneness; it states that accidents do not only happen randomly but rather some people are more prone to have an accident. Later research has not obtained conclusive evidence either for or against this theory, arguing that people can go through more accident-prone periods according to their psychological state.

In 1931, Heinrich developed the domino theory, proposing that a sequence of factors led to accidents. These factors were mostly focused on the person, and how they are influenced by personal mistakes combined with dangerous or unsafe behavior. This behavior causes the accident, which ends up in injury or property damage. Heinrich (1931) postulated that if dangerous or unsafe behavior was removed, then accidents could be prevented. The domino theory was modified by Adams (1976), focusing not on personal characteristics, but on properties of the organization. Adams (1976) suggested that it was the organizational structure that determines the occurrence of operational errors, which are the cause of incidents or accidents. Bird and Germain (1985) specified that accidents had "multiple sources." In other words, there are many causes that can explain an accident; therefore, identifying sources will avoid accidents. This idea is the basis of the studies that try to identify the factors behind the accident, finding that multiple variables affect the outcome.

Later, Howell et al. (2002) proposed a completely different theory based on cognitive systems engineering. These authors highlight that previous approaches do not take into account factors such as the nature and dynamics of work on the construction site. Individual and organizational pressures push workers into hazardous 
113 conditions. Howell et al. (2002) argue that there is a safe area in which workers

114 perform their work, bounded by the pressures of economic failure of the organization,

115 personal exertion and acceptable performance. These external pressures can make

116 the worker start working in the area where there is a loss of control.

117 Through the years, the occurrence of accidents has gone from being considered a

118 random phenomenon to being the result of a series of factors that are possible to

119 determine and control. Meanwhile, the main cause of accidents stopped being the

120 person as an individual, or his/her characteristics or the company itself, to a much more

121 complex scenario, in which there are multiple sources at the individual and

122 organizational level that can cause accidents (Bird \& Germain, 1985). Therefore,

123 identifying the main factors affecting safety performance in projects has been a goal for

124 researchers and practitioners over many years and not only in the construction

125 industry. Since the nineties, there have been many studies that attempt to identify the

126 practices that are most effective in reducing accidents. Most of these studies have

127 been based on surveys or case studies considering the preventive activities performed

128 in construction projects. Table 1 shows a historical overview of the most relevant

129 studies and practices identified so far, as well as the data collection method employed.

\begin{tabular}{|c|c|c|c|c|c|c|c|c|c|c|c|c|c|c|}
\hline CONTRIBUTION & Year & $\mathbf{A}$ & B & C & D & $\mathbf{E}$ & $F$ & $\mathbf{G}$ & $\mathbf{H}$ & I & $\mathbf{J}$ & $\mathbf{K}$ & $\mathbf{L}$ & Data Collection \\
\hline Levitt and Parker & 1976 & $\mathrm{X}$ & $\mathrm{X}$ & $\mathrm{X}$ & & & $\mathrm{X}$ & & & & $\mathrm{X}$ & & & Survey \& Interviews \\
\hline Hinze and Harrison & 1981 & & $\mathrm{X}$ & $X$ & & & $\mathrm{X}$ & & & & & & & Case Study \\
\hline Samelson and Levitt & 1982 & $\mathrm{X}$ & $x$ & $X$ & & $X$ & $X$ & & $X$ & $X$ & $\mathrm{X}$ & & & Survey \\
\hline Hinze and Raboud & 1988 & & $\mathrm{X}$ & & & & X & $\mathrm{X}$ & & & & & & Interviews \\
\hline Hinze and Figone & 1988 & & $\mathrm{X}$ & & & & X & & & $X$ & & $X$ & & Survey \\
\hline Liska et al. & 1993 & $\mathrm{X}$ & $\mathrm{X}$ & $X$ & $\mathrm{X}$ & $\mathrm{X}$ & & & & & & & & Survey \\
\hline Jaselskis et al. & 1996 & $x$ & $\mathrm{X}$ & $x$ & $x$ & $\mathrm{X}$ & $\mathrm{X}$ & $\mathrm{X}$ & & $X$ & & $\mathrm{X}$ & & Survey \& Empirical Data \\
\hline Harper and Koehn & 1998 & $\mathrm{X}$ & $\mathrm{X}$ & & & & & & $\mathrm{X}$ & & $\mathrm{X}$ & & & Case Study \\
\hline Sawacha et al. & 1999 & & $x$ & $X$ & & & $X$ & $\mathrm{X}$ & & $X$ & $x$ & & & Survey \\
\hline Hinze and Wilson & 2000 & $\mathrm{X}$ & $x$ & $X$ & $\mathrm{X}$ & $\mathrm{X}$ & & & & & & & & Survey \\
\hline Hinze & $2002 a$ & $X$ & $\mathrm{X}$ & $X$ & $\mathrm{X}$ & X & $\mathrm{X}$ & $\mathrm{X}$ & $\mathrm{X}$ & $\mathrm{X}$ & & & & Survey \& Case Study \\
\hline Hinze & $2002 b$ & & & $x$ & & & & & & & & & & Survey \\
\hline Mohamed & 2002 & $X$ & $X$ & & & & X & $\mathrm{X}$ & $X$ & & & & & Survey \\
\hline Fang et al. & 2004 & & $X$ & & & & $X$ & & & & $\mathrm{X}$ & $\mathrm{X}$ & $X$ & Survey \\
\hline Tam et al. & 2004 & $\mathrm{X}$ & $\mathrm{X}$ & & & & X & & & & $\mathrm{X}$ & & & Survey \\
\hline Fung et al. & 2005 & & $X$ & & & & $X$ & & $X$ & & $\mathrm{X}$ & & & Survey \\
\hline Teo et al. & 2005 & & $\mathrm{X}$ & $X$ & & & X & & $X$ & $X$ & & & & Survey \\
\hline Abudayyeh et al. & 2006 & & $\mathrm{X}$ & $X$ & & & X & & $X$ & & & & & Survey \\
\hline Huang and Hinze & 2006 & $\mathrm{X}$ & $X$ & & $\mathrm{X}$ & & $x$ & & & & & $X$ & & Survey \\
\hline Razuri et al. & 2007 & $x$ & $x$ & $X$ & $\mathrm{X}$ & $\mathrm{X}$ & $X$ & $\mathrm{X}$ & & $X$ & & & $X$ & Survey \\
\hline Aksorn and Hadikusumo & 2008 & $\mathrm{X}$ & $\mathrm{X}$ & $\mathrm{X}$ & & & $x$ & & $X$ & & $\mathrm{X}$ & & & Survey \\
\hline Hallowell and Gambatese & 2009 & $\mathrm{X}$ & $\mathrm{X}$ & $X$ & $\mathrm{X}$ & $\mathrm{X}$ & $X$ & $\mathrm{X}$ & $X$ & $X$ & & & & Delphi Method \\
\hline Pellicer and Molenaar & 2009 & $x$ & $\mathrm{X}$ & & & $\mathrm{X}$ & & & & & & & $\mathrm{X}$ & Discussion \\
\hline Vinodkumar and Bhasi & 2010 & $\mathrm{x}$ & $X$ & & & & X & & $X$ & & $X$ & $x$ & & Survey \\
\hline Hallowell and Calhoun & 2011 & $x$ & $X$ & & $\mathrm{X}$ & X & $X$ & $\mathrm{X}$ & $X$ & $\mathrm{X}$ & & $x$ & & Delphi Method \\
\hline
\end{tabular}




\begin{tabular}{|c|c|c|c|c|c|c|c|c|c|c|c|c|c|c|}
\hline CONTRIBUTION & Year & $\mathbf{A}$ & B & $\mathbf{C}$ & D & $\mathbf{E}$ & $\mathbf{F}$ & $\mathbf{G}$ & $\mathrm{H}$ & I & $\mathbf{J}$ & $\mathbf{K}$ & $\mathbf{L}$ & Data Collection \\
\hline Lai et al. & 2011 & & $\mathrm{X}$ & $\mathrm{X}$ & & & $\mathrm{X}$ & $\mathrm{X}$ & $\mathrm{X}$ & & & & & Survey \\
\hline Hallowell & 2012 & $X$ & $x$ & & & $X$ & & & & $X$ & & $X$ & $X$ & Case Study \\
\hline Bridi et al. & 2013 & $x$ & $x$ & $X$ & & & & $\mathrm{X}$ & $X$ & & & & & Survey \\
\hline Hinze et al. & 2013 & $X$ & $\mathrm{X}$ & $X$ & $\mathrm{X}$ & X & $X$ & $X$ & $X$ & $\mathrm{X}$ & & & & Survey \\
\hline Hallowell et al. & 2013 & $x$ & $x$ & $X$ & $x$ & $x$ & $x$ & $X$ & $X$ & $x$ & & & & Case Study \\
\hline Olutuase & 2014 & $X$ & $X$ & & & & & $\mathrm{X}$ & $X$ & & $X$ & $X$ & & Survey \\
\hline Yorio and Wachter & 2014 & $X$ & $x$ & & & $\mathrm{X}$ & & $\mathrm{X}$ & $X$ & & & & & Survey \& Empirical Data \\
\hline Wachter and Yorio & 2014 & $X$ & $X$ & & & $x$ & & $\mathrm{X}$ & $X$ & & & & & Survey \& Empirical Data \\
\hline Guo et al. & 2015 & & $x$ & $X$ & & & $X$ & & $X$ & $X$ & & & & Grounded Theory \\
\hline Wu et al. & 2015 & & $\mathrm{X}$ & $x$ & & & $X$ & & $X$ & & $\mathrm{X}$ & & & Survey \\
\hline Guo and Yiu & 2016 & $\mathrm{X}$ & $\mathrm{X}$ & $\mathrm{X}$ & & & $x$ & & $X$ & $X$ & $\mathrm{X}$ & & & Interviews \\
\hline
\end{tabular}

\section{Table 1. Literature review}

NOTES:

B. Safety orientation and specialized training

C. Evaluation and reward

D. Drug and alcohol testing

E. Accident and incident investigation

F. Management commitment

G. Staffing for safety

H. Worker involvement

I. Subcontract management

J. Safety equipment

K. Safety audits

L. Management safety training

One of the most relevant papers in the field was published by Jaselskis et al. (1996) who proposed specific practices to improve safety at the project and company level. The additional merit of this work is that it used empirical data, such as recordable incident rates (or accident rates) and experience modification rates. Using a survey of corporate safety coordinators, these authors found that the most significant practices were: upper-management attitude, project-management team turnover, time devoted to safety by field safety representatives, formal and informal safety meetings with supervisors, specialty sub-contractors, site safety inspections, and worker safety performance penalties.

Later, a research report issued by the Construction Industry Institute identified the five practices with the greatest impact in reducing accidents (Liska et al., 1993; Hinze \& Wilson, 2000): pre-project and pre-task planning, safety orientation and specialized training, evaluation and reward, drug and alcohol testing, and accident and incident investigation. Later on, Hinze (2002a) extended these five techniques to nine, adding the following: management commitment, staffing for safety, worker involvement, and subcontractor management.

Besides these nine factors that are widely recognized among researchers and practitioners (Hallowell et al., 2013; Hinze et al., 2013), three additional ones have 
been included in the literature review (see Table 1): safety equipment, safety audits, and management safety training. In 1999, Sawacha and colleagues carried out a survey of construction workers in the United Kingdom; they concluded that the supply and use of safety equipment was among the top five most effective practices (Sawacha et al., 1999). Similar studies, such as the ones developed by Fang et al. (2004), Tam et al. (2004), Fung et al. (2005), Vinodkumar \& Bhasi (2010), and Wu et al. (2015) corroborated this finding. Regarding safety audits and inspections, Jaselskis et al. (1996) considered them to be key recommended practices; this proposal was later supported by Fang et al. (2004), Huang \& Hinze (2006), Vinodkumar \& Bhasi (2010), Hallowell (2012), and Olutuase (2014), among others.

Training of upper management in safety issues is not a common practice among contributors; however, the authors of this research have added it because of personal conviction of its importance. Pellicer \& Molenaar (2009) stated the key importance of education and training for engineering managers, especially in the construction industry, and how it influences the safety culture. Even though most of the authors analyzed proposed training up to the supervisor level, as described in Guo \& Yiu (2016), just a few (Razuri et al., 2007; Hallowell, 2012) have taken into consideration training of the managers; Fang et al. (2004) measured the hours of safety education per year for a manager, including it as a main factor regarding safety education in construction.

After reviewing the most relevant literature in the field, very few contributions regarding safety management practices implemented by construction companies deal with empirical studies that relate these practices to better safety performance. Jaselskis et al. (1996) were pioneers in this matter, setting the course. However, it was not until recently that Yorio \& Wachter (2014) and Wachter \& Yorio (2014) developed an empirical study of safety management practices based on the accident rate as well as the days away, restricted duty, or job transfer rate. Furthermore, the authors of this paper have found few studies that analyze a combination of practices, instead of the 
effect of individual practices. Therefore, the analysis of combination of practices using empirical data is the point of departure of this research.

\section{Materials and Methods}

As stated in the Introduction, the goal of this paper is to detect the best performing combinations of practices or strategies for different sizes of companies. The authors have used data from the Safety Mutual of the Chilean Chamber of Construction (Safety Mutual hereafter) as their source for empirical data. The Safety Mutual is a non-profit organization that provides medical insurance and technical assistance on safety management to companies in all industries. It includes more than 2,600 workers as well as more than 50 support centers. The Safety Mutual invests in prevention to diminish occupational accidents, mainly through programs specifically requested by and designed for companies, which include: certificates of safety compliance, audits, specific assessments, and training courses and workshops, among others. The Safety Mutual has data from companies (practices implemented and accident rates), but it does not really know the actual on-site impact of these practices.

The Safety Mutual's database included data of construction companies and prevention practices carried out every year; it was also possible to obtain information such as company size, building indicators, and other data to characterize the companies involved. However, it was difficult and complex to process and analyze data from the original database because the system was designed to store data, not to assess information. Significant efforts were made to improve the quality of the data, because the original data was input by safety experts from each of the companies who might have different criteria for defining prevention practices. Also, companies may have carried out other activities that the Safety Mutual was not aware of, and hence those activities would not have been included in the database. To build the database used in the analysis, the following activities were carried out: 
- Filter companies by category, in order to analyze only the construction companies in the Safety Mutual: a total of 1,180 companies.

- Sort companies by size of business: four levels considering the number of employees.

- Calculate the rate of accidents per year for each company.

- Select four years with a total of 4,506 records.

The next step was to identify the prevention practices undertaken each year by each company. There were a total of 221 different prevention practices; due to the fact that each one of these practices was defined by the safety experts using his/her own style, a homogenization process was needed. This way, the next step was to group prevention practices into categories to simplify the analysis. Considering the literature review, carried out in advance and summarized in the previous Section (see Table 1), as well as the classification of activities performed by the Safety Mutual, the research team decided to classify the 221 practices into seven categories of practices, as follows:

1. Accidents \& Incidents Investigation: activities related to the capture of information of accidents and incidents.

2. Safety Planning \& Resources: activities carried out by safety staff (such as the preparation of safety plans) as well as activities related with safety equipment that workers should use.

3. Management Commitment: activities that demonstrate the willingness and commitment to safety from management, which otherwise would not be carried out.

4. Workers' Safety Training: activities such as courses, workshops, seminars, and all kind of safety training for workers.

5. Management Safety Training: similar to the previous group, but focused on the company management.

6. Audits \& Certifications: regular activities performed by the Safety Mutual. 
7. Safety Incentives \& Rewards: all kinds of recognition for good safety records.

It can be noted that the first four categories concur with some of the best practices proposed by Hinze (2002a). The last three, however, were adjusted to the characteristics of many practices developed and encouraged by the Safety Mutual, which are focused on training of managers, certifications, and safety incentives. As indicated previously, the objective of this research is to identify which of these practices are most effective.

Eighty strategies, or combination of categories of practices, were detected in the database. However, only the ones with at least 30 available records were taken into consideration for the analysis, obtaining a total of 14 strategies. The main metric to compare the effectiveness of these strategies was the accident rate, defined as shown in Eq. 1.

$$
\text { Accident rate }=\frac{\text { number of accidents }}{\text { average labor force }}
$$

Summarizing, the final database comprised: original descriptive variables (year, average number of employees, number and description of practices implemented out of the original 221 practices), additional descriptive variables (size of the company, and presence or absence of any practice belonging to a category defined in the previous classification), and response variable (accident rate). With this data, the research team performed the following analyses:

- Preliminary analysis of practices (221), categories (7), and all strategies (80).

- Descriptive analysis of the 14 most frequently implemented strategies, measuring the incremental added value of different categories in different scenarios.

- Classification tree of the 14 most frequently implemented strategies, using the exhaustive CHAID (Chi-Squared Automatic Interaction Detector) algorithm and displaying a routing graph of strategies. This statistical analysis was carried out using IBM SPSS Statistics (version 20.0). 


\section{Analysis and Discussion of Results}

\subsection{Preliminary Analysis}

Considering the 221 original practices coded by the Safety Mutual, a visual analysis of the number of practices implemented versus the accident rate per year and a broad dispersion of data, which may explain the low correlation.

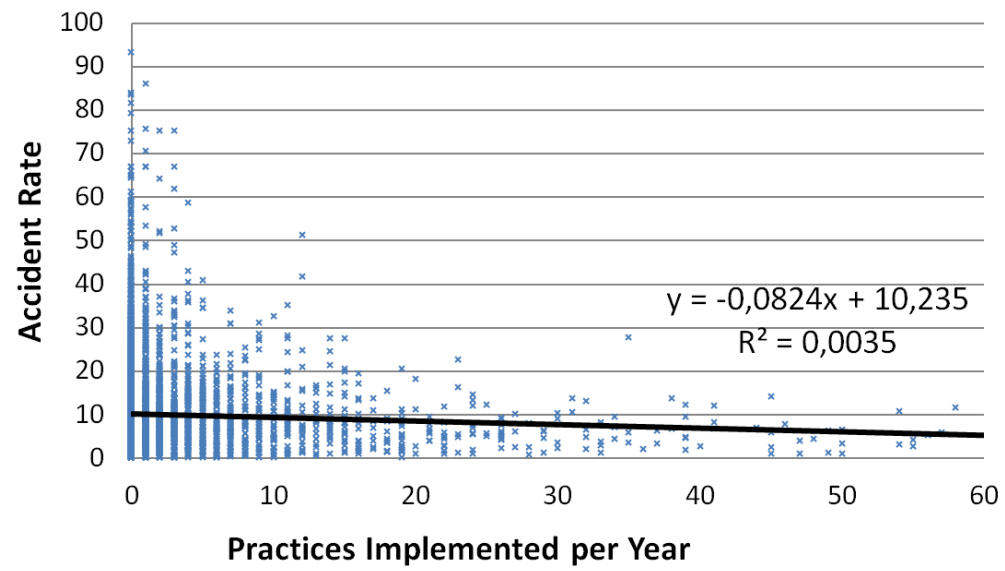

Figure 1. Practices implemented per year versus accident rate

Further analysis comprises a two sample z-test in order to determine if two population means are equal (in this case referring to the accident rate), considering that the variance of the population is known and, furthermore, the population is normally distributed (with more than 30 records each). First, this analysis is applied to 292 groups of companies (taken from the population in the database) that have 293 implemented intervals of practices: none, one or two, three to seven, eight to ten, or 
more than eleven. Table 2 shows the results of the analysis of accident rates for these groups of practices. A two sample z-test is applied to determine if two populations' means are equal; the comparison is performed between a group and its following successor in size considering the fewer necessary amounts of additional practices to get a statistically significant difference between the accident rates of both groups $(0$ practices compared to 1-2 practices, and so on). The results displayed in Table 2 prove that there is a statistically significant difference between the different groups; therefore, this corroborates the previous visual analysis of Figure 1: the more practices implemented, the lower the accident rate.

\begin{tabular}{lccccc}
\hline & 0 practices & $\mathbf{1 - 2}$ practices & $\mathbf{3 - 7}$ practices & $\mathbf{8 - 1 0}$ practices & >11 practices \\
\hline Mean (accident rate) & 13.20 & 11.89 & 10.88 & 9.04 & 7.71 \\
Variance (known) & 148.50 & 115.78 & 90.76 & 51.21 & 43.96 \\
Observations & 2,065 & 717 & 543 & 123 & 300 \\
z & 2.71 & 1.76 & 2.41 & 1.78 & \\
p $(Z \leq z)$ one tail & 0,00 & 0.04 & 0.01 & 0.04 & \\
z critical value & 1.64 & 1.64 & 1.64 & 1.64 & \\
\hline
\end{tabular}

$304 \quad$ Table 2. Results of z-test for accident rates according to different number of practices implemented in the company

In a similar way, an analysis of means can be performed for the quantity of practices performed, grouping companies according to their size: micro (9 or less employees), small (between 10 and 49), medium (between 50 and 199), and large 310 companies (200 or more). A two sample z-test is also used to decide if two populations' 311 means are equal; in this case, the comparison is performed between a group and its 312 following successor in size (micro-companies to small companies, and so on). Results 313 are displayed in Table 3 . The quantity of practices (mean) shows a statistically significant difference between the different sizes of companies. In this case, the larger

315 the company, the more practices implemented. 


\begin{tabular}{lcccc}
\hline & Micro-companies & Small companies & Medium companies & Large companies \\
\hline Mean (quantity of practices) & 0.18 & 0.68 & 1.91 & 9.04 \\
Variance (known) & 0.56 & 4.83 & 14.04 & 237.67 \\
Observations & 510 & 1,623 & 1,482 & 891 \\
z & -7.81 & -11.03 & -13.58 & 0.00 \\
p(Zsz) one tail & 0.00 & 0.00 & 1.64 & \\
z critical value & 1.64 & 1.64 & & \\
\hline
\end{tabular}

$318 \quad$ Table 3 . Results of z-test for quantity of practices performed according to different size of company

The next step is focused on the seven categories of practices, which come from 322 grouping the 221 original practices in the database. These categories were sorted 323 using a tornado diagram in Figure 2. This chart displays, in the vertical axis, the seven categories of practices. In the horizontal axis, the graph shows the difference between

325 the accident rate for a company implementing (blue color) and not implementing (red 326 color) a category, compared to the average accident rate (in vertical), which in this 327 case is 10.01. Figure 2 shows that there is indeed a difference in the accident rate of companies that perform some categories of practices compared to those that do not

329 perform them; this is applicable to all categories except Accidents \& Incidents 330 Investigation. Companies that do not implement any of the other six variables have (on average) a higher accident rate than the mean of all records (10.01). The companies

332 that implement the category Safety Incentives \& Rewards outperform the others, 333 decreasing the accident rate by more than $25 \%$. Regarding the other five categories, 334 the differences are not so significant. Furthermore, there are also small differences for 335 companies that do not implement practices, except in the case of Accidents \& Incidents 336 Investigation whose difference with the average is negative. 


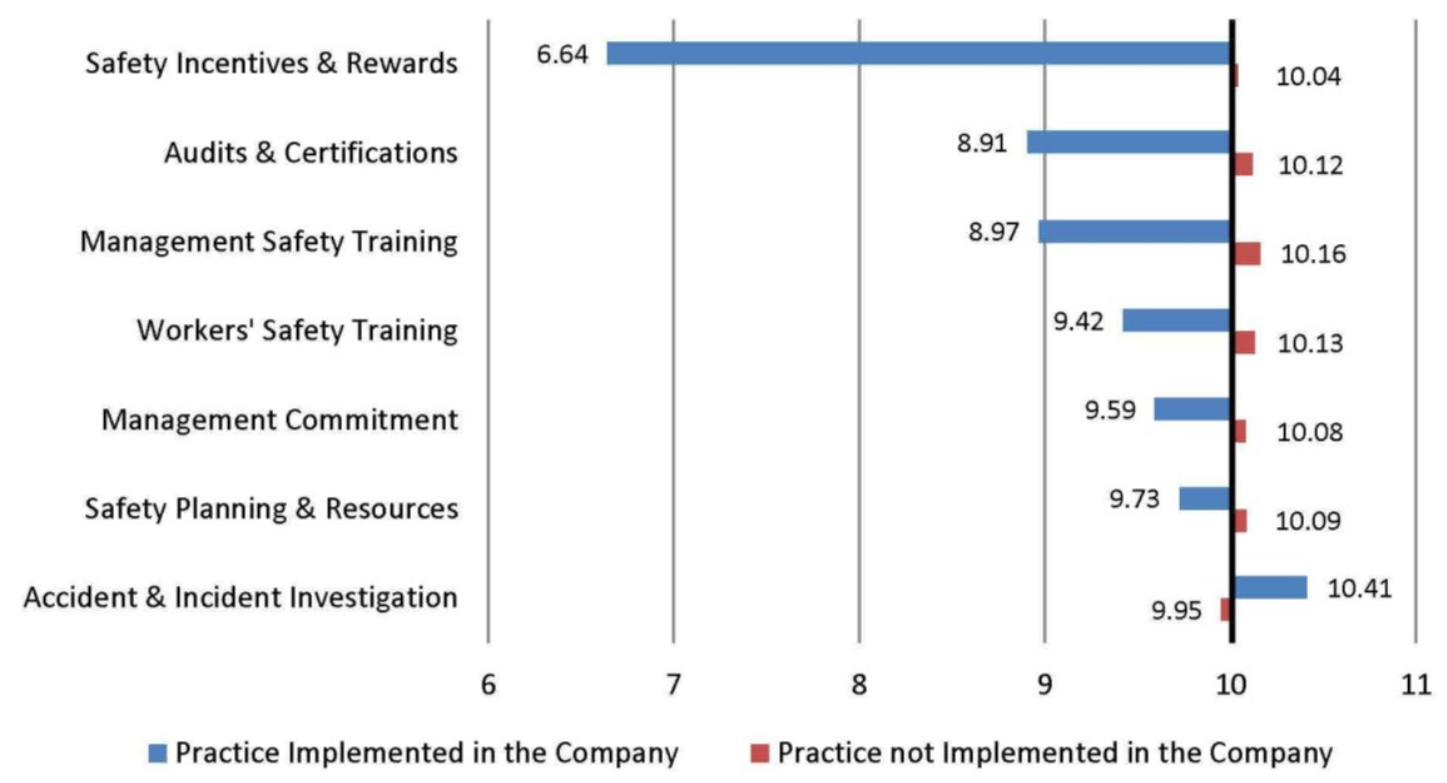

Figure 2. Accident rate considering if the category is implemented or not

The percentage of companies conducting prevention practices ranges from $0.8 \%$

340 to $19.9 \%$ of the total for each of the seven categories. The impact of prevention can be

341 considered by dividing the average accident rate of the companies that do not 342 implement a specific variable by the average accident rate of the companies that do 343 implement that category; this is the effectiveness index proposed by Razuri et al. 344 (2007) displayed in Figure 3. The effectiveness index reveals that companies carrying 345 out prevention practices are always a smaller portion compared with companies that do 346 not perform these practices. 


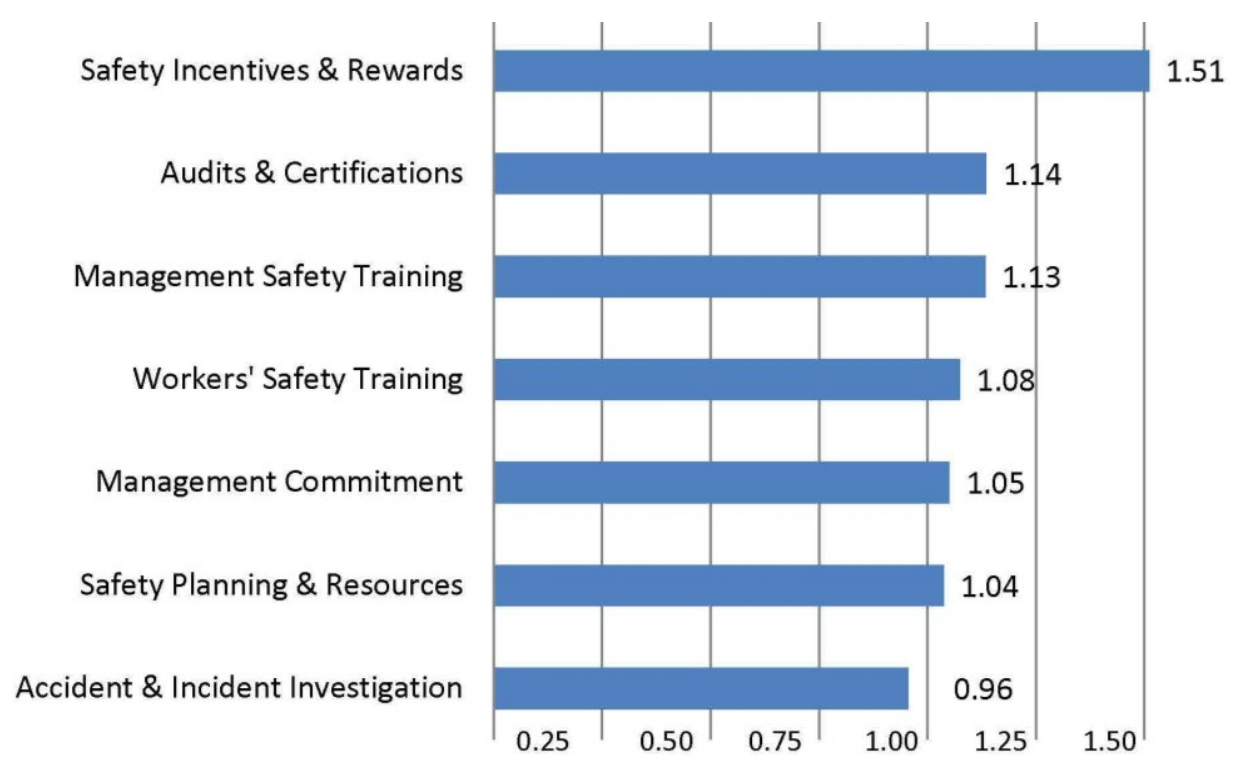

Figure 3. Effectiveness index of prevention categories

Safety Incentives \& Rewards is the category that is most effective, from the point of view of the accident rate. Companies that do not implement any practice in this category have an accident rate $51 \%$ higher than companies that do implement this category, as shown in Figure 3. This conclusion contradicts some previous contributions stating that incentives are counterproductive (Hinze, 2002b), or may be effective in the short term, but counterproductive in the long-term (Guo et al., 2015). identified for further analysis. These combinations of practices are called "strategies" 
from now on. Figure 4 shows 80 different strategies (or combinations of practices) found in the database, ordered in a tornado graph form, in a similar way as in Figure 2; the 80 strategies (in horizontal) are classified according to the difference between their accident rate compared to the average accident rate (in vertical), from highest (left) to lowest (right). As seen in Figure 4, there is a good fit to the linear equation proposed. However, accident rates among categories are not normally distributed.

Figure 4. Tornado diagram of accident rate variation for each strategy

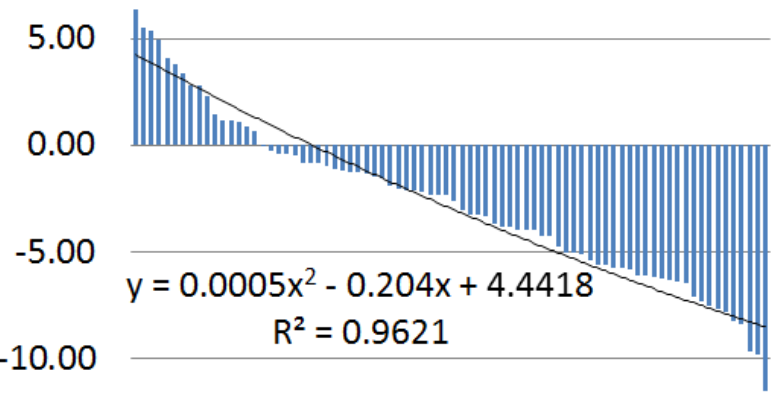

$-15.00$

Distance from the Average Accident Rate to each Category

Linear Fit

Figure 5 shows that as the number of preventive practices increases (shown as a percentage in the right side of the diagram), accident rates decrease (displayed as a ratio in the left side). However, this relationship shows high variability. It seems that the number of preventive practices "per se" has limited influence on accident rates; furthermore, individual practices may have different effectiveness too. Therefore, more important than the number of practices is the right combination of them (or strategy). 


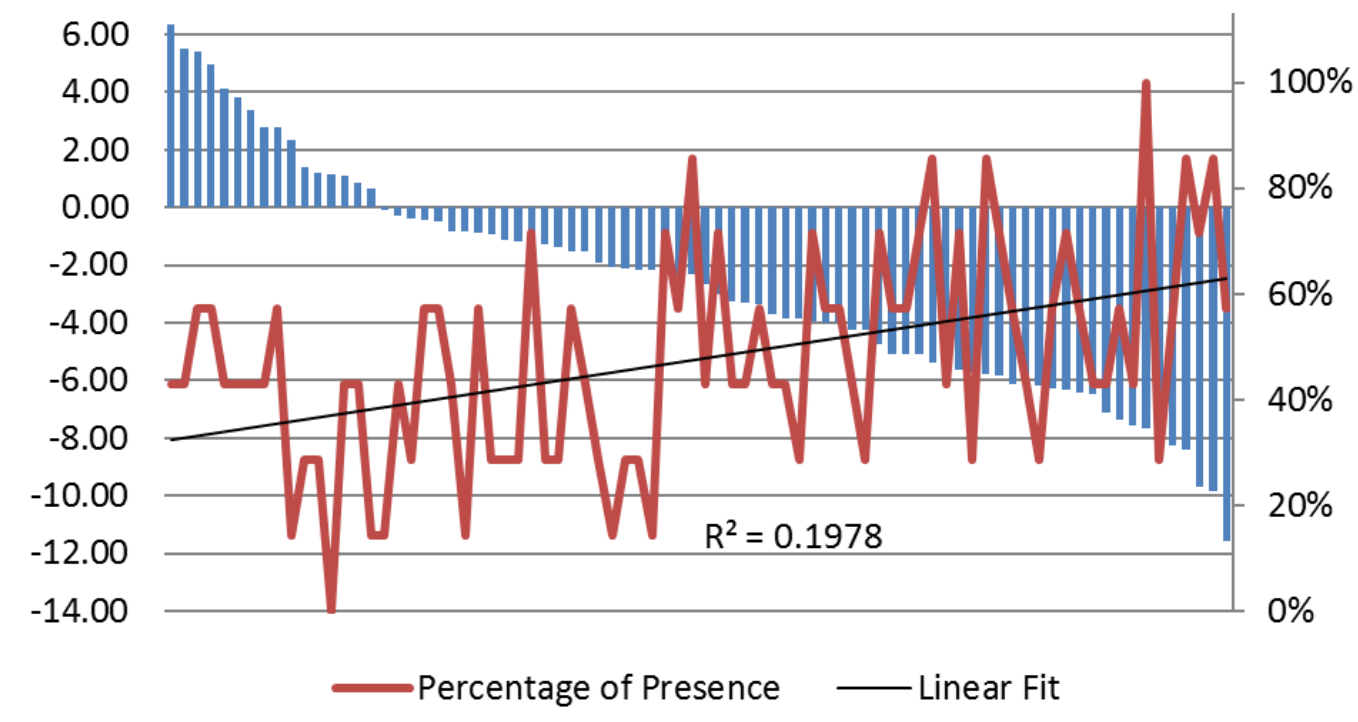

Figure 5. Relative accident rate and presence of practices

\subsection{Descriptive Analysis of Strategies}

In order to increase the soundness of the results, only those strategies with 30 or more available records were considered in this analysis; therefore, only 14 strategies were analyzed. Figure 6 allows for a global review of these 14 strategies. The upper part of Figure 6 displays the chosen 14 strategies (in the horizontal axis); the vertical axis shows not only the average accident rate of each strategy (left), but also the percentage of the presence of the seven categories in that particular strategy (right). The lower part of Figure 6 exhibits the chosen 14 strategies (in horizontal), and the seven categories used for grouping the practices as well as the distance to the average accident rate, record count and percentage of records (in vertical). 


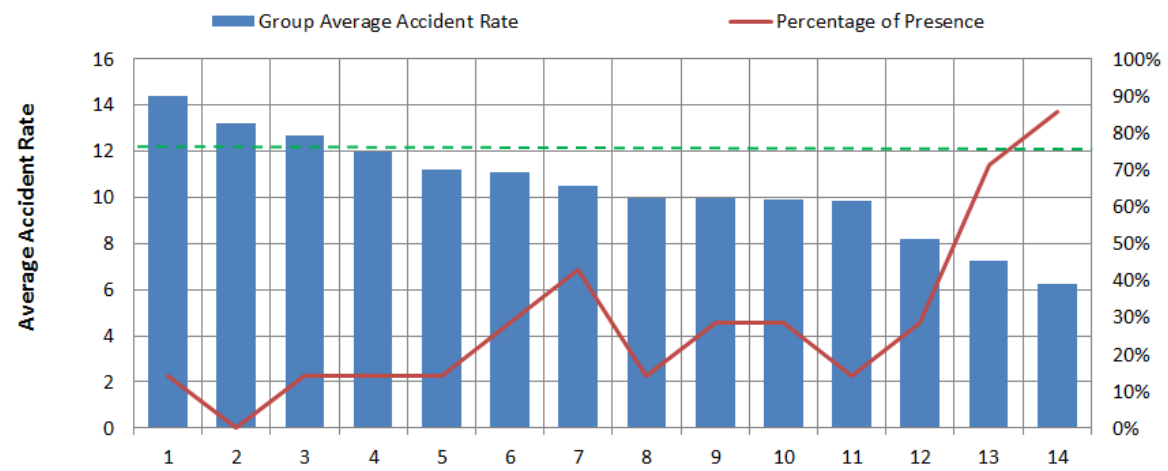

\begin{tabular}{|l|r|r|r|r|r|r|r|r|r|r|r|r|r|r|}
\hline Accident \& Incident Investigation & YES & NO & NO & NO & NO & YES & YES & NO & NO & NO & NO & NO & YES & YES \\
\hline Safety Planning \& Resources & NO & NO & YES & NO & NO & YES & YES & NO & YES & NO & NO & YES & YES & YES \\
\hline Management Commitment & NO & NO & NO & NO & NO & NO & YES & YES & YES & NO & NO & NO & YES & YES \\
\hline Workers' Safety Training & NO & NO & NO & NO & YES & NO & NO & NO & NO & YES & NO & NO & YES & YES \\
\hline Management Safety Training & NO & NO & NO & YES & NO & NO & NO & NO & NO & YES & NO & YES & YES & YES \\
\hline Audits \& Certifications & NO & NO & NO & NO & NO & NO & NO & NO & NO & NO & YES & NO & NO & YES \\
\hline Safety Incentives \& Rewards & NO & NO & NO & NO & NO & NO & NO & NO & NO & NO & NO & NO & NO & NO \\
\hline Percentage of Presence & $14 \%$ & $0 \%$ & $14 \%$ & $14 \%$ & $14 \%$ & $29 \%$ & $43 \%$ & $14 \%$ & $29 \%$ & $29 \%$ & $14 \%$ & $29 \%$ & $71 \%$ & $86 \%$ \\
\hline Group Average Accident Rate & 14.36 & 13.20 & 12.67 & 11.97 & 11.19 & 11.08 & 10.50 & 9.99 & 9.94 & 9.88 & 9.87 & 8.18 & 7.27 & 6.25 \\
\hline Distance to Average Accident Rate & $19 \%$ & $10 \%$ & $5 \%$ & $-1 \%$ & $-7 \%$ & $-8 \%$ & $-13 \%$ & $-17 \%$ & $-17 \%$ & $-18 \%$ & $-18 \%$ & $-32 \%$ & $-40 \%$ & $-48 \%$ \\
\hline Record Count & 85 & 2066 & 199 & 67 & 320 & 45 & 37 & 64 & 47 & 33 & 44 & 43 & 36 & 70 \\
\hline Percentage of Records & $2.3 \%$ & $55.1 \%$ & $5.3 \%$ & $1.8 \%$ & $8.5 \%$ & $1.2 \%$ & $1.0 \%$ & $1.7 \%$ & $1.3 \%$ & $0.9 \%$ & $1.2 \%$ & $1.1 \%$ & $1.0 \%$ & $1.9 \%$ \\
\hline
\end{tabular}

Figure 6. Strategies with at least 30 records

Figure 6 highlights some facts; for example, even though these 14 strategies represent $84.2 \%$ of the records in the database, the top nine strategies comprise an average of $1.2 \%$ of records in the database. It is worthy to note that the second worst strategy (no category implemented) is widely adopted and accounts for $55 \%$ of total records; however, it has twice the accident rate of the best strategy. Maybe the most striking fact is that the category Safety Incentives \& Rewards is not present in any of the strategies analyzed, being seldom used by companies; even though the high impact of this category was already previously analyzed, the scarce data so far impedes considering it for the configuration of the strategies.

In general, the two most effective strategies (those with the two lowest accident rates) have more categories implemented (five and six, respectively). The most popular strategy (second-worst performance) does not have any of the seven categories implemented. This confirms previous results that indicate that the higher the number of prevention activities, the lower the accident rates. However, this trend presents interesting features. For example, strategies 6, 9 and 12 have two categories each; one 
415 of the categories is Safety Planning \& Resources, and the strategies vary only in the

416 presence of the practices Accidents \& Incidents Investigation, Management

417 Commitment, and Management Safety Training, respectively. Only with this difference,

418 strategy 6 has an average accident rate of 11.08 , strategy 9 of 9.94 , and strategy 12 of

419 8.18. With an equal number of categories, there are differences of up to $26 \%$ in the accident rate, highlighting the importance of the strategy itself.

Using a similar approach, it is possible to assess the impact of individual categories of practices. Strategies 1, 3, 4, 5, 8 and 11 (Figure 6) have only one category each. They are displayed in Figure 7 in order to analyze their individual impact, compared with companies that do not do anything. Figure 7 is arranged similar to Figure 6. Companies that only implemented the category Accidents \& Incidents Investigation had accident rates higher than companies without any category of practice. This is consistent with the definition of the practice; as explained previously, serious accidents, to determine their causes. In that sense, it can be defined as a practice of reaction rather than prevention.

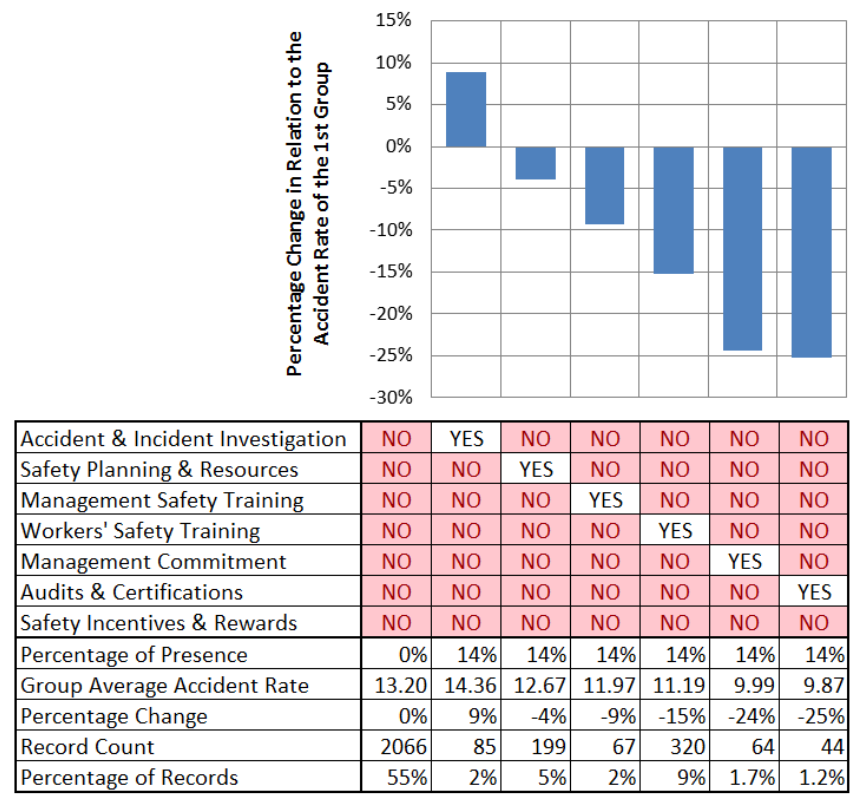

Figure 7. Analysis of the impact of individual categories 
Safety Planning \& Resources had an impact of $4 \%$ on the accident rate compared

with companies without any category implemented. As in the previous case, this is because many of the practices that comprise this category correspond to the preparation of plans and safety programs, which are just the first steps to reduce the number of accidents in a workplace. Management Safety Training had a 9\% impact on the accident rate, whereas the category Workers Safety Training reduced the accident rate by more than $15 \%$. This shows that training focused on those who carry out the activities has a higher impact than training focused on those who manage them.

Finally, the categories of practice Management Commitment and Audits \& Certifications reduced accident rates by approximately $25 \%$. This significant reduction can be explained in part because companies that adopt this type of commitment from upper management have a low accident rate, and they work to reach the zero accidents target, with a strategic vision of the company. It is noteworthy that in the category Audits \& Certifications there are individual practices that lead to certification of the company, which is a clear indication that the company has safety as one of its strategic goals.

In summary, this analysis identified categories of practices and strategies that have a greater impact and can support the design of more effective and economical safety management strategies. One of the greatest potentials of this analysis is that it allows inputting a categorical variable; the sample can be divided into two nodes, according to the presence or absence of the category, and that generates the combinations of variables that make up each strategy. With this, the impact of each of these strategies can be measured, in general, but also the impact of each of these strategies in companies that have different characteristics, such as the type of project, size of company, etc. For example, Figure 8 shows the impact of the different strategies on the accident rate (in horizontal) according to company size (in vertical, left); smaller firms 


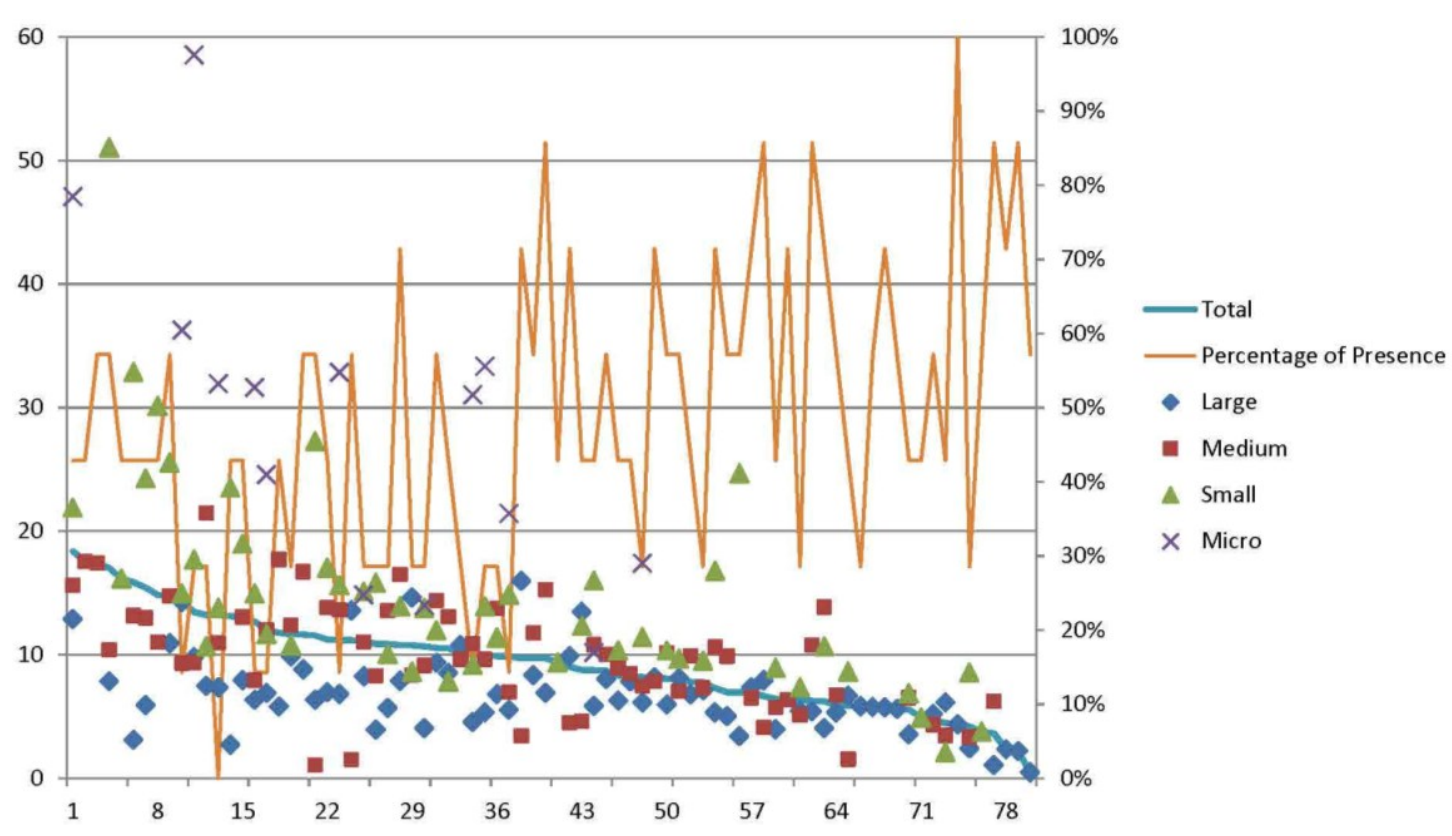

Figure 8. Accident rate of each strategy analyzed by company size

have a larger dispersion and worse results for the same strategies than larger firms, whereas larger companies have much lower dispersion than smaller firms.

\subsection{Classification Tree Approach}

\section{2}

4

As a method to support the design of optimal strategies, categories of practices can be identified that, combined with others already in place, minimize the accident rate. The aim is to get the best combination of categories (independent variables or predictors) that explain the following output (dependent or predicted variable), to answer this question: Does the accident rate decrease when applying a specific category of practice? This procedure can be systematized using the classification tree method, implementing the exhaustive CHAID (Chi-Squared Automatic Interaction Detector) algorithm (Kass, 1980; Biggs et al., 2011). Because the answer to the previous question is categorical dicothomic (yes/no), this algorithm uses a Chi-Squared test in order to divide the data into two groups (nodes), which have a statistically significant difference between the average accident rates of both nodes. Data is 
systematically split into separate groups (nodes) in a way that the variation of the

477 dependent variable is minimized within the groups and maximized among the groups

478 (Ramaswami and Bhaskaran, 2010); the process is repeated until no statistically significant difference is found (stopping rule). This will not only make sure that there are statistical differences between the accident rate for different nodes, but also allows the identification of the order of application of the categories, which in turn allows the measurement of the marginal impact of each of these different combinations.

This method is displayed using a tree diagram, which allows visualizing the relationship between the dependent and independent variables in a graphical way, as shown in Figure 9. It is a tree of 12 nodes, with seven terminal nodes (which means seven paths), using five categories (independent variables) out of the total of seven possible categories. Figure 9 includes not only the mean and standard deviation, but also the percentage of presence for each of the categories that the algorithm used in each node. The seven terminal nodes represent $100 \%$ of all records in the database.

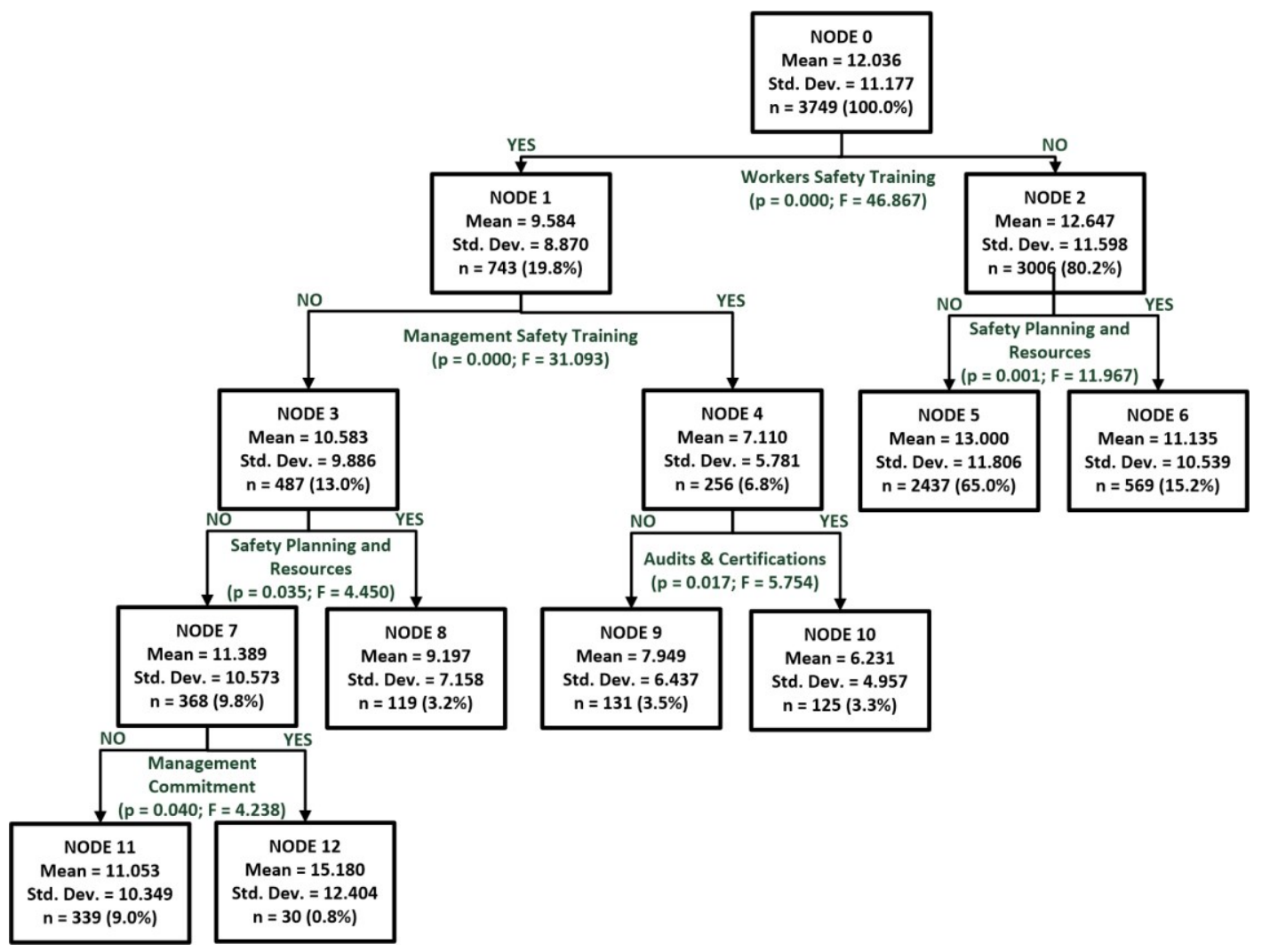

Figure 9. Classification tree using exhaustive CHAID algorithm 
In order to get a better interpretation of the data obtained from the CHAID analysis,

492 Figure 10 is displayed. The numbers in the upper horizontal of Figure 10 are the 493 terminal nodes in Figure 9. As in Figures 6 and 7, in the graph (vertical) part of Figure 49410 , the record count and percentage of records is shown, whereas, in the table 495 (vertical) the distance to the average accident rate is included; furthermore, this table in 496 Figure 10 includes not only the five categories used by the CHAID algorithm, but also 497 the two additional ones (Accidents \& Incidents Investigation as well as Safety Incentives \& Rewards) specifying their percentage of presence.

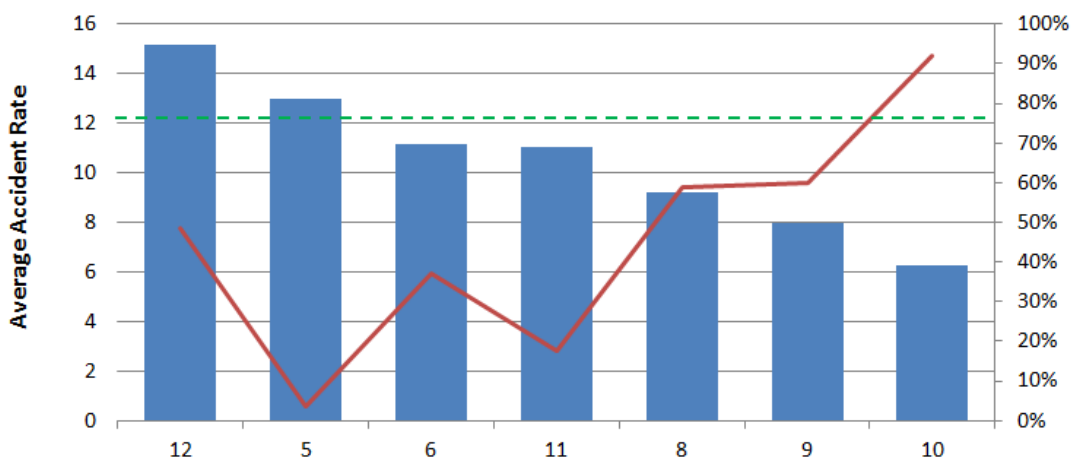

\begin{tabular}{|l|c|c|c|c|c|c|c|}
\hline Accident \& Incident Investigation & $63 \%$ & $7 \%$ & $33 \%$ & $4 \%$ & $54 \%$ & $43 \%$ & $70 \%$ \\
\hline Safety Planning \& Resources & NO & NO & YES & NO & YES & $60 \%$ & $94 \%$ \\
\hline Management Commitment & YES & $6 \%$ & $36 \%$ & NO & $55 \%$ & $53 \%$ & $83 \%$ \\
\hline Workers' Safety Training & YES & NO & NO & YES & YES & YES & YES \\
\hline Management Safety Training & NO & $5 \%$ & $29 \%$ & NO & NO & YES & YES \\
\hline Audits \& Certifications & $23 \%$ & $3 \%$ & $21 \%$ & $2 \%$ & $36 \%$ & NO & YES \\
\hline Safety Incentives \& Rewards & $3 \%$ & $0 \%$ & $2 \%$ & $0 \%$ & $7 \%$ & $4 \%$ & $6 \%$ \\
\hline Percentage of Presence & $48 \%$ & $4 \%$ & $37 \%$ & $18 \%$ & $59 \%$ & $60 \%$ & $92 \%$ \\
\hline Group Average Accident Rate & 15.18 & 13.00 & 11.14 & 11.05 & 9.20 & 7.95 & 6.23 \\
\hline Distance to Average Accident Rate & 3.14 & 0.96 & -0.90 & -0.98 & -2.84 & -4.09 & -5.80 \\
\hline Record Count & 30 & 2437 & 569 & 338 & 119 & 131 & 125 \\
\hline Percentage of Records & $0.8 \%$ & $65.0 \%$ & $15.2 \%$ & $9.0 \%$ & $3.2 \%$ & $3.5 \%$ & $3.3 \%$ \\
\hline Node & 12 & 5 & 6 & 11 & 8 & 9 & 10 \\
\hline
\end{tabular}

Figure 10. Average accident rate terminal nodes with exhaustive CHAID algorithm

The best strategy corresponds to node 10 (Workers' Safety Training + Management Safety Training + Audits \& Certifications); this is the final node with lowest accident rate mean in Figure 9. This strategy also has the most presence of each of the other four categories and also for the whole set of categories (92\%). 
However, only $3.3 \%$ of the records contain this strategy. The other two best strategies, corresponding to nodes 8 and 9, get similar results. Their first category is Workers' Safety Training; node 9 considers later Management Safety Training, whereas node 8 considers Safety Planning \& Resources instead. The former gets more distance to the accident rate, as well as slightly more presence of the whole set $(60 \%)$ and similar percentage records (3.5\%). Anyway, Workers' Safety Training is present in these three best strategies.

Figures 9 and 10 also indicate that the worst strategy represents only $0.8 \%$ of total records, and it has a high percentage of categories present (48\%); maybe companies with very bad performance decided to implement many prevention activities in order to improve their outcomes quickly. Due to this fact, as well as the low percentage of records, this category may not be illustrative. The second worst strategy represents $65 \%$ of the records, but no category out of the whole set was chosen by the algorithm in this strategy. This strategy also has a very low presence of the total categories (4\%), whereas the most successful strategy (lowest accident rate mean) has a presence of $92 \%$. This confirms the analysis of Figure 6 : the higher the percentage of categories implemented in the strategy, the lower the accident rate. However, as in the previous analysis, this growth is not completely linear; a node may have a higher presence of practices, but still have worse accident rates than another node, and vice versa. This relationship shows again that, for intermediate ranges, the right combination is more important than the number of practices.

\section{Conclusions}

The analysis of the data showed that even though the number of prevention activities had a statistically significant correlation with the accident rate (the more practices implemented the less the accident rate), the analysis in the graphical strategy format showed that this factor itself provided only a partial explanation. Significant 
differences occurred once a certain number of practices were reached; at a given point, the marginal contribution of adding more prevention practices is virtually zero. In future work the authors will explore this relation for companies of different characteristics.

The analysis of the combined effect of prevention practices (safety management strategies) showed that the choice of the right combination of practices was more important than just the number of practices implemented. In this research, the most optimized combined strategy implements practices in three categories: Workers' Safety Training, Management Safety Training, and Audits \& Certifications; this strategy gets the lowest accident rate, with the most presence of each of the other four categories and also for the whole set of categories. It is worth noting that Workers' Safety Training is always present in the best three strategies analyzed; hence, it seems to have a key role in any prevention strategy. Furthermore, Safety Incentives \& Rewards is the category that is most effective, from the point of view of the accident rate, even though it was very scarcely implemented in the dataset. Finally, practices related to Accidents \& Incidents investigation are reactive, generally used as an aftermath of an accident and, therefore, may not be a good indicator of performance.

This analysis approach can bring significant improvements to companies by contributing to the design of effective strategies that can lead to best results at a minimum cost. Similarly, the Safety Mutual can benefit themselves and their associates by developing highly effective prevention strategies with the least cost. Moreover, these methods can help to determine the order of implementation of different practices, so as to achieve greater impact at first, and then continue with higher marginal impact.

The strategy analysis method proposed seems to be an attractive method to design safety management strategies if the appropriate data is available. Because of the space constraints, a feature that was not discussed in this paper is that the method can be used to perform an individualized analysis for a company, considering its particular characteristics: company size, type of business, type of projects, or any other attribute or combination. This eventually allows the development of custom designed 
programs. When applied in companies which have massive data on their prevention programs, this method could enable them to measure the impact on safety for every new management initiative. This method also allows analyzing individual factors of a safety program, or any unconventional prevention activity to be implemented, provided that there are sufficient data for the analysis. Future work will pursue the development of software that integrates data capture with this methodology to support the design of safety management strategies for companies and projects according to their specific characteristics and needs.

There are some limitations to this research. First, the data regarding implementation of practices was reported to the Chilean Safety Mutual by the companies; therefore, the quality of the data is related to the quality of each organization's self-reporting. Moreover, regarding the safety practices and their combination, frequency is not the only condition to take into consideration. The quality of implementation may also affect the outcome. Another issue to consider is the magnitude of the accidents: a major one has greater impact than many minors. These limitations can lead to future lines of research.

The method used in this research can be applied to other countries. The categories are defined according to an in-depth literature review and they can be equally valid in other contexts. Nevertheless, the results cannot be extrapolated to other scenarios unless previous analyses have been developed to check if their safety culture is similar to that of the Chilean construction industry.

\section{Acknowledgments}

The authors are grateful to the management and staff of the Safety Mutual of the Chilean Chamber of Construction for the insight and help provided during this research. The authors also thank Ms. Lisa Gingles for revising the manuscript as well as three anonymous reviewers for their valuable suggestions. 


\section{References}

Abudayyeh, O., Fredericks, T. K., Butt, S. E., and Shaar, A. (2006). An investigation of management's commitment to construction safety. International Journal of Project Management, 24(2), 167-174, http://doi.org/10.1016/j.ijproman.2005.07.005.

Adams, E. E. (1976). Accident causation and the management system. Professional Safety, October, 26-29.

Aksorn, T., and Hadikusumo, B. H. W. (2008). Critical success factors influencing safety program performance in Thai construction projects. Safety Science, 46(4), 709-727, http://doi.org/10.1016/j.ssci.2007.06.006.

Biggs, D., De Ville, B., and Suen, E. (1991). A method of choosing multiway partitions for classification and decision trees. Journal of Applied Statistics, 18(1), 49-62.

Bird, F., and Germain, G. (1990). Practical loss control leadership, ILCI Press, Logansville, CA.

Bridi, M.E., Pellicer, E., Fabro, F., Viguer, M.E., Echeveste, M.E.S., and Formoso, C.T. (2013). Identificação de práticas de gestão da SST em obras de construção civil. Revista do Ambiente Construído, 13(3), 43-58.

Fang, D. P., Xie, F., Huang, X. Y., and Li, H. (2004). Factor analysis-based studies on construction workplace safety management in China. International Journal of Project Management, 22(1), 43-49, http://doi.org/10.1016/S0263-7863(02)001151.

Fung, I. W. H., Tam, C. M., Tung, K. C. F., and Man, A. S. K. (2005). Safety cultural divergences among management, supervisory and worker groups in Hong Kong construction industry. International Journal of Project Management, 23(7), 504512, http://doi.org/10.1016/j.ijproman.2005.03.009. 
Greenwood, M., and Woods, H. M. (1919). The incidence of industrial accidents upon individuals with special reference to multiple accidents. HM Stationery Office, No. 4, London.

Guo, B.H., and Yiu, T.W. (2016). Developing leading indicators to monitor the safety conditions of construction projects. Journal of Management in Engineering, 32(1), $04015016-1 / 14$

Guo, B.H., Yiu, T.W., and Gonzalez, V.A. (2015). Identifying behaviour patterns of construction safety using system archetypes. Accident Analysis \& Prevention, 80, $125-141$

Hallowell, M. R. (2012). Safety-knowledge management in american construction organizations. Journal of Management in Engineering, 28(2), 203-211, http://doi.org/10.1061/(ASCE)ME.1943-5479

Hallowell, M. R., and Calhoun, M. E. (2011). Interrelationships among highly effective construction injury prevention strategies. Journal of Construction Engineering and Management, 137(11), 985-993, http://doi.org/10.1061/(ASCE)CO.19437862.0000354 .

Hallowell, M. R., and Gambatese, J. A. (2009). Construction safety risk mitigation, Journal of Construction Engineering and Management, 135(12), 1316-1323, http://doi.org/10.1061/(ASCE)CO.1943-7862.0000107

Hallowell, M. R., Hinze, J. W., Baud, K. C., and Wehle, A. (2013). Proactive construction safety control: measuring, monitoring, and responding to safety leading indicators. Journal of Construction Engineering and Management, 139(10), 04013010-1/8, http://doi.org/10.1061/(ASCE)CO.1943-7862.0000730.

Harper, R. S., and Koehn, E. (1998). Managing industrial construction safety in southeast texas. Journal of Construction Engineering and Management, 124(6), 452-457, http://doi.org/10.1061/(ASCE)0733-9364(1998)124:6(452).

Heinrich, H. (1931) Industrial accident prevention. McGraw-Hill, New York. 
Hinze, J. (2002a) Making zero accidents a reality. Cll Research Rep. 160-11, University of Texas at Austin, EEUU.

Hinze, J. (2002b). Safety incentives: do they reduce injuries? Practice Periodical on Structural Design and Construction, $\quad 7(2), \quad 81-84$. http://doi.org/10.1061/(ASCE)1084-0680(2002)7:2(81)

Hinze, J., and Figone, L. A. (1988). Subcontractor safety as influenced by general contractors on small and medium sized projects. Source Document 38, Constr. Industry Inst., Univ. of Washington, Seattle, Wash.

Hinze, J., and Harrison, C. (1981). Safety programs in large construction firms. Journal of the Construction Division ASCE, 107(3), 455-467.

Hinze, J., and Raboud, P. (1988). Safety on large building construction projects. Journal of Construction Engineering and Management, 114(2), 286-293.

Hinze, J., and Wilson, G. (2000). Moving toward a Zero Injury Objective, Journal of Construction Engineering and Management, 126 (5), 399-403.

Hinze, J., Hallowell, M., and Baud, K. (2013). Construction-safety best practices and relationships to safety performance. Journal of Construction Engineering and Management, 139(10), 04013006-1/8, http://doi.org/10.1061/(ASCE)CO.1943$\underline{7862.0000751 .}$

Howell, G., Ballard, G., Abdelhamid, T., and Mitropoulos, P. (2002). Working near the edge: a new approach to construction safety. Proceedings of the 10th Annual Conference of the International Group for Lean Construction, Gramado, Brasil.

Huang, X., and Hinze, J. (2006). Owner's role in construction safety. Journal of Construction Engineering and Management, 132(2), 164-173.

Jaselskis, E. J., Anderson, S. D., and Russell, J. S. (1996). Strategies for achieving excellence in construction safety performance. Journal of Construction Engineering and Management, 122(1), 61-70.

Kass, G.V. (1980), An exploratory technique for investigating large quantities of categorical data. Applied Statistics, 29(2), 119-127. 
Lai, D. N. C., Liu, M., \& Ling, F. Y. Y. (2011). A comparative study on adopting human resource practices for safety management on construction projects in the United States and Singapore. International Journal of Project Management, 29(8), 10181032, http://doi.org/10.1016/j.jproman.2010.11.004

Levitt, R. E., and Parker, H. W. (1976). Reducing construction accidents-top management's role. Journal of the Construction Division ASCE, 102(3), 465-478.

Liska, R. W., Goodloe, D., and Sen, R. (1993). Zero accident techniques. Source Document 86, Constr. Industry Inst., Univ. of Texas at Austin, Tex.

Mohamed, S. (2002). Safety climate in construction site environments. Journal of Construction Engineering and Management, 128(5), 375-384, http://doi.org/10.1061/(ASCE)0733-9364(2002)128:5(375).

Olutuase, S.O. (2014). A study of safety management in the Nigerian construction industry. IOSR Journal of Business and Management, 16(3), 1-10.

Pellicer, E., and Molenaar, K.R. (2009). Discussion of 'Developing a model of construction safety culture' by Rafiq M. Choudhry, Dongping Fang, and Sherif Mohamed. Journal of Management in Engineering, 25(1), 44-45.

Pellicer, E., Carvajal, G.I., Rubio, M.C., and Catalá, J. (2014). A method to estimate occupational health and safety costs in construction projects. KSCE Journal of Civil Engineering, 18(7), 1955-1965.

Razuri, C., Alarcon, L.F. and Diethelm, S. (2007), Evaluating the effectiveness of safety management practices and strategies in construction practices. Proceedings IGLC-15, July 2007, Michigan, 271-281.

Ramaswami, M., Bhaskaran, R. (2010). A CHAID based performance prediction model in education data mining, International Journal of Computer Science Issues, 7(1), 10-18.

Samelson, N. M., and Levitt, R. E. (1982). Owner's guidelines for selecting safe contractors. Journal of the Construction Division ASCE, 108(4), 617-623. 
Sawacha, E., Naoum, S., \& Fong, D. (1999). Factors affecting safety performance on construction sites. International Journal of Project Management, 17(5), 309-315, http://doi.org/10.1016/S0263-7863(98)00042-8.

Tam, C. M., Zeng, S. X., \& Deng, Z. M. (2004). Identifying elements of poor construction safety management in China. Safety Science, 42(7), 569-586, http://doi.org/10.1016/j.ssci.2003.09.001

Teo, E. A. L., Ling, F. Y. Y., \& Chong, A. F. W. (2005). Framework for project managers to manage construction safety. International Journal of Project Management, 23(4), 329-341, http://doi.org/10.1016/j.jproman.2004.09.001.

Vinodkumar, M. N., \& Bhasi, M. (2010). Safety management practices and safety behaviour: Assessing the mediating role of safety knowledge and motivation. Accident Analysis \& Prevention, 42(6), 2082-2093, http://doi.org/10.1016/j.aap.2010.06.021.

Wachter, J.K., and Yorio, P.L. (2014). A system of safety management practices and worker engagement for reducing and preventing accidents: An empirical and theoretical investigation. Accident Analysis \& Prevention, 68, 117-130.

Waehrer, G. M., Dong, X. S., Miller, T., Haile, E., and Men, Y. (2007). Cost of occupational injuries in construction in the United States. Accident Analysis and Prevention, 39(6), 1258-1266.

Wu, X., Liu, Q., Zhang, L., Skibniewski, M. J., and Wang, Y. (2015). Prospective safety performance evaluation on construction sites. Accident Analysis \& Prevention, 78, 58-72.

Yorio, P.L., and Wachter, J.K. (2014). The impact of human performance focused safety and health management practices on injury and illness rates: Do size and industry matter? Safety Science, 62, 157-167. 